TRABAJOS ORIGINALES

Rev Obstet Ginecol Venez. 2021; 81 (4): 354-364.

https://doi.org/10.51288/00810408

\title{
Utilidad del recuento de plaquetas en el diagnóstico del cáncer de ovario
}

\author{
(D) Linder Mariano Díaz Colmenarez, ${ }^{1,2}$ (D) Belkys Carolina Zambrano Ramón, ,2 \\ (D) Daniel Alejandro Omaña Carrero, ${ }^{1,3}$ (iD Manuel Santos Luque, Ph.D. ${ }^{1,4}$
}

\begin{abstract}
RESUMEN
Objetivo: Evaluar la utilidad del recuento plaquetario y el CA-125 en la discriminación entre tumores ováricos malignos y benignos en el Hospital Universitario de Los Andes, en un periodo de 5 años.

Métodos: Investigación observacional retrospectiva. Se revisaron 419 historias de pacientes codificadas como tumores ováricos. Se utilizó como prueba estándar de oro el reporte anatomopatológico definitivo. Se calcularon múltiples parámetros estadísticos de rendimiento diagnóstico desde tablas 2 x 2 y se graficó curvas de características operativas del receptor.

Resultados: El recuento plaquetario en las pacientes con tumores ováricos malignos invasivos fue de 386/nl (IC 95\%: 362409), en tumores benignos fue 243/nl (IC $95 \%$ : 235-251) y en tumores borderline 237/nl (IC 95\%: 198-276). Aunque el área bajo la curva características operativas del receptor fue mayor para el recuento plaquetario en comparación con los niveles de CA-125 (0,880 vs 0,790) esta diferencia no fue estadisticamente significativa. Entre los tumores mucinosos, los malignos no presentaron CA-125 elevado, pero si recuento plaquetario elevado. El valor predictivo positivo más alto del recuento plaquetario fue en posmenopáusicas 95,6\% para $>350 / \mathrm{nl}$ y $100 \%$ para $>400 / \mathrm{nl}(\mathrm{p}<0,001)$.

Conclusión: El recuento plaquetario parece presentar utilidad similar a CA-125 para discriminar tumores malignos de
\end{abstract} benignos y pudiera mejorar el rendimiento diagnóstico al combinarse ambos valores preoperatorios.

Palabras clave: Plaquetas, Trombocitosis, Ovario, Tumor, Cáncer, CA-125, Exactitud diagnóstica, Menopausia.

Usefulness of the platelet count in the diagnosis of the ovarian cancer

SUMMARY

Objective: To evaluate the usefulness of the platelet count and CA-125 in the discrimination between malignant and bening ovarian tumors at the Hospital Universitario de Los Andes, in a period of 5 years.

Methods: Retrospective observational research. 419 patient medical stories coded as ovarian tumors were reviewed. The definitive pathology report was used as the gold standard test. Multiple statistical parameters of diagnostic performance were calculated from $2 \times 2$ tables and receiver operating characteristic (ROC) curves were plotted.

Results: The mean platelet count: with invasive malignant ovarian tumors was 386/nl (CI $95 \%$ 362-409), in benign tumors it 243/nl (CI $95 \%$ 235-251) and in borderline tumors 237/nl (CI $95 \%$ 198-276). Although the area under the curve receiver operating characteristic was higher for platelet count compared to CA125 levels (0.880 vs 0.790) this difference was not statistically significant. Among the mucinous tumors, the malignant ones did not present an elevated CA-125, but an elevated platelet count. Highest PPV of the platelet count was $95.6 \%$ for $>350 / \mathrm{nl}$ in postmenopausal women and $100 \%$ for $>400 / n l(p<0.001)$.

Conclusion: The platelet count seems to have a similar utility to CA-125 to discriminate malignant from benign tumors and could improve diagnostic performance when both preoperative values are combined.

Keywords: Platelets, Thrombocytosis, Ovary, Tumor, Cancer, CA-125, Diagnostic accuracy, Menopause.

\begin{abstract}
${ }^{1}$ Especialista en Obstetricia y Ginecología. Universidad de Los Andes. Mérida, Venezuela. ${ }^{2}$ Centro de Ultrasonografía AGB, Clínica Sanatorio Alemán, Concepción, Chile. ${ }^{3}$ Unidad Clínica La Magdalena. Barrancabermeja, Colombia. ${ }^{4}$ Ginecólogo Oncólogo. Instituto Autónomo Hospital Universitario Los Andes. Mérida, Venezuela. Correo de correspondencia: ginecologo.obstetra@gmail.com
\end{abstract}

Forma de citar este artículo: Díaz Colmenarez L M, Zambrano Ramón B C, Omaña Carrero DA, Santos Luque M. Utilidad del recuento de plaquetas en el diagnóstico del cáncer de ovario. Rev Obstet Ginecol Venez. 2021; 81(4):354-364. https://doi.org/10.51288/00810408

\section{INTRODUCCIÓN}

Una adecuada identificación del carácter y comportamiento tumoral ovárico es indispensable para determinar si el manejo y tratamiento inicial debe llevarse a cabo en unidades de ginecología oncológica, 
mejorando la sobrevida o si por el contrario puede manejarse de manera conservadora (1). El escenario opuesto, es decir, considerar un tumor benigno como cáncer puede condicionar derivaciones innecesarias a centros especializados con sus correspondientes consecuencias económicas, psicológicas y legales (2, $3)$.

La detección de cáncer ovárico en estadios avanzados determina un impacto en la sobrevida de las pacientes: la detección en el estadio I implica una sobrevida a 5 años del 93,3\%, 1,5 veces más que si se diagnostica en estadio II, casi 4 veces más que estadio III y hasta 7 veces más oportunidades de vida que si se diagnosticara en estadio IV (4).

Se han propuesto múltiples estrategias diagnósticas para la detección precoz de malignidad en tumores ováricos, sin embargo, aún no hay consenso para tal fin (5).

Se ha descrito una importante relación entre concentración plaquetaria y cáncer ovárico: al disminuir el recuento de plaquetas, administrando anticuerpos antiplaquetarios, también disminuye el tamaño tumoral y su capacidad de propagación, de forma similar, las dimensiones tumorales aumentan $140 \%$ ante una transfusión plaquetaria (6). Existe evidencia biomolecular (7) de que las plaquetas desempeñan un rol fundamental en la atracción, adhesión y comportamiento biológico de las células cancerígenas del ovario lo que explicaría, en parte, la diseminación y probable respuesta terapéutica. Por otro lado, se ha reportado una menor hiperreactividad proagregación plaquetaria en muestras in vitro provenientes de pacientes con cáncer ovárico vs tumores benignos, lo que plantea que la relación entre plaquetas y cáncer ovárico parece obedecer a funciones no hemostáticas (8).

En 1992 (9) se publica, por primera vez, un estudio de masas pélvicas (la gran mayoría tumores ováricos), en el cual reportan que la trombocitosis $(>400000 /$ $\mathrm{mm}^{3}$ ) tiene utilidad para discriminar entre malignas y benignas, con especificidad y valor predictivo positivo similar al antígeno carbohidratado 125 (CA-125). Además, la combinación de ambas pruebas logró mejorar aún más estos parámetros estadísticos. En los últimos 28 años, múltiples autores han publicado el papel del recuento plaquetario como valor pronóstico en cáncer ovárico, sin embargo, realizando una minuciosa búsqueda por múltiples índices bibliográficos, bases de datos y buscadores (PubMed/ MEDLINE, Web of Science, Scopus, Embase, Google Scholar, Scielo, ResearchGate), sin límite de idioma, solo se encontraron 6 publicaciones (10-15) que han estudiado el rol del recuento plaquetario preoperatorio como discriminador entre malignidad y benignidad ovárica, ninguna de estas en español, así como tampoco en población latinoamericana.

El propósito del estudio fue evaluar, con mayor detalle a lo reportado, la utilidad del recuento plaquetario para discriminar entre tumores ováricos malignos y benignos, comparándose a su vez con niveles de CA125 como marcador sérico tradicional.

\section{MÉTODOS}

Se realizó una investigación de tipo descriptiva y retrospectiva de casos y controles, en el Instituto Autónomo Hospital Universitario Los Andes (IAHULA) de Mérida, Venezuela, en un periodo de 5 años continuos. La muestra se obtuvo desde la base de datos detallada de todos los tumores de ovario tratados durante un quinquenio y utilizada previamente en otras investigaciones $(16,17)$. Conservándose confidencialidad para los datos de identificación, se analizó el estado menopaúsico, el recuento plaquetario y los niveles séricos prequirúrgicos de CA-125, el reporte definitivo anatomopatológico $\mathrm{y}$, finalmente, el estadio según la Federación Internacional de Ginecología y Obstetricia (FIGO). 
Se utilizaron los siguientes criterios de inclusión: 1) patología tumoral de ovario, 2) intervenidas quirúrgicamente y 3) reporte definitivo de anatomía patológica de lesión ovárica. Fueron excluidas aquellas pacientes con: 1) patología oncológica simultánea en otra localización, 2) tratamiento en otra institución médica y que el conocimiento en el instituto fuese por una recaída del cáncer ovárico, 3) paciente embarazada al momento del diagnóstico, 4) falta de resultados preoperatorios de recuento plaquetario y/o CA-125, 5) enfermedad infecciosa simultánea, 6) enfermedad hematológica actual o previa, 7) patología reumatológica conocida, 8) disfunción renal y/o hepática.

Se consideraron casos los tumores malignos y controles los benignos. Para fines analíticos, los de bajo potencial de malignidad (TBPM) fueron incluidos en el primer grupo. El reporte definitivo de anatomía patológica fue la prueba "estándar de oro". El punto de corte para CA125 fue de $35 \mathrm{U} / \mathrm{L}$. Se definió trombocitosis al conteo plaquetario > 350/nanolitro (nl). Los valores para ambas determinaciones se obtuvieron dentro de las 72 horas previas a la cirugía. Se consideró menopausia en pacientes $\geq 45$ años y con $\geq 12$ meses de amenorrea o en mujeres histerectomizadas $\geq 50$ años. Para el análisis estadístico se utilizaron números absolutos, porcentajes, medias, desviación estándar, intervalos de confianza del $95 \%$ (IC95\%) en las variables según el programa SPSS versión 22,0 para Windows ${ }^{\circledR}$ (IBM, Chicago, IL, USA), también se utilizó Excel 2010 (Microsoft Office, Redmond, Washington, USA). Se graficó la curva características operativas del receptor (ROC) y se calcularon las respectivas áreas bajo la curva (AUC). Se determinó desde tablas 2x2: sensibilidad (S), especificidad (E), valor predictivo positivo (VPP) y negativo (VPN), likelihood ratio positivo (LR+) y negativo (LR-), exactitud diagnóstica (ED) y odds ratio diagnóstica (DOR). Los resultados se expresaron en tablas y figuras.

Se utilizó la prueba de Chi cuadrado $\left(\mathrm{X}^{2}\right)$ con corrección de Pearson o test exacto de Fisher cuando ambas variables fueron cualitativas; al analizar una variable cualitativa con cuantitativa: T de Student y para el análisis de variables cualitativas con más de 2 categorías junto a variable cuantitativa, test de ANOVA. Se utilizó prueba U de Mann-Whitney en distribuciones no paramétricas. Se consideró valores significativos estadísticamente cuando $\mathrm{p}<0,05$.

Se recibió autorización para el estudio por el comité de investigación del hospital. Se utilizó guía STARD para elaboración del manuscrito (18).

\section{RESULTADOS}

De un total de 419 pacientes codificadas como tumores de ovario, finalmente se analizaron 247 , siendo de estos un $34,82 \%$ malignos ( 86 ) y un $65,18 \%$ benignos (161). Las exclusiones se exponen en la Figura 1.

Las edades de las pacientes oscilaron entre 14 años y 81 años, con una media de 40,7 años $\pm 15,1$ años. La media de edad con tumor maligno fue de 46,6 \pm 14,4 años y con tumor benigno de $37,6 \pm 14,6$ años ( $p$ $<0,001)$. Dentro de los malignos, la edad media fue: $49,5 \pm 12,3$ años en epiteliales vs $21,3 \pm 6,3$ años en germinales $(\mathrm{p}<0,0001)$.

Entre los tumores de ovario, el 77,67 \% fue de origen epitelial, el $19,54 \%$ origen germinal y el 1,86\% de los cordones sexuales/estroma.

De aquellos malignos, el 88,37\% (76/86) fueron de origen epitelial, un $9,30 \%(8 / 86)$ de origen germinal, y los restantes se originaron de cordones sexuales/ estroma e indiferenciados. Los serosos destacaron entre los malignos derivados del epitelio, con un 76,31\% (58/76), seguido en frecuencia por endometroides y mucinosos: 10,52 \% (8/76) y 5,26 \% (4/76), respectivamente, otros subtipos histopatológicos sumaron un $7,91 \%(6 / 76)$. Se presentaron 3 
Figura 1. Flujograma STARD de pacientes estudiadas.

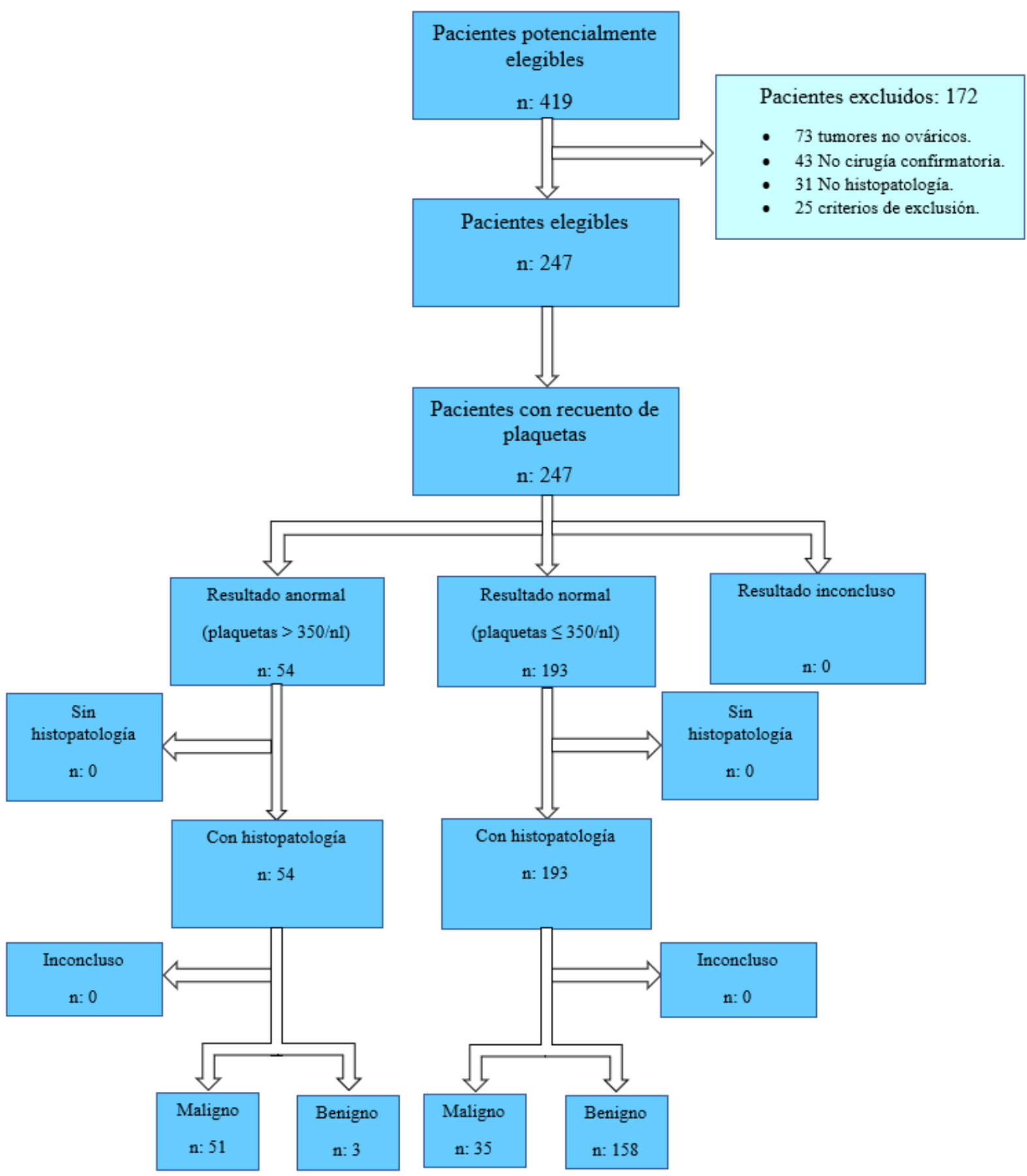

tumores mixtos: siendo todos disgerminoma + seno endodérmico en un mismo ovario.
La distribución de los tumores según la histopatología $\mathrm{y}$ el valor medio del recuento plaquetario y el CA- 
125 se presentan en Tabla $1(\mathrm{p}<0,001)$. La Tabla 2 $(\mathrm{p}<0,001)$ muestra el valor medio de recuento plaquetario y CA-125 para tumores malignos según estadio y estado menopáusico. Los cánceres epiteliales tipo II según etiopatogenia con un 52,63\% (40/76).
En el cálculo de rendimiento diagnóstico se utilizó puntos de corte de 350/nl o 400/nl para plaquetas y $35 \mathrm{U} / \mathrm{ml}$ para CA-125 (Tabla 3), como puntos de corte tradicionalmente utilizados. La Figura 2 y la tabla 3 permiten observar a detalle el rendimiento diagnóstico del recuento plaquetario y del CA-125.

Tabla 1. Distribución según histopatología y medias de plaquetas y de CA-125.

\begin{tabular}{|c|c|c|c|c|}
\hline HISTOPATOLOGIA & $\mathrm{n}$ & $(\%)$ & $\overline{\mathrm{x}}$ plaquetas (IC95 \%) & $\overline{\mathrm{x}}$ CA-125 (IC95\%) \\
\hline TBPM seroso & 5 & 2 & $241(192-290)$ & $13,50(0,63-26,36)$ \\
\hline TBPM mucinoso & 1 & 0,4 & 215 & 36,20 \\
\hline Adenocarcinoma seroso & 53 & 21,5 & $389(360-417)$ & $228,63(101,89-355,37)$ \\
\hline Adenocarcinoma mucinoso & 3 & 1,2 & $363(215-510)$ & $19,05(15,19-23,00)$ \\
\hline Adenocarcinoma endometrioide & 8 & 3,2 & $414(348-480)$ & $155,15(45,61-264,40)$ \\
\hline Adenocarcinoma células claras & 1 & 0,4 & 376 & 78,10 \\
\hline Carcinoma Brenner & 1 & 0,4 & 197 & 45,00 \\
\hline Carcinoma epidermoide & 2 & 0,8 & $345(205-485)$ & $153,05(152,41-153,68)$ \\
\hline Carcinoma Indiferenciado & 2 & 0,8 & $363(0-726)$ & $113,20(28,40-198,00)$ \\
\hline Tumor neuroendocrino carcinoide & 1 & 0,4 & 267 & 13,00 \\
\hline Teratoma inmaduro & 2 & 0,8 & $351(239-462)$ & $22,35(5,19-39,50)$ \\
\hline Disgerminoma & 2 & 0,8 & $329(0-658)$ & $120,70(0-241,00)$ \\
\hline Tumor Seno endodérmico & 1 & 0,4 & 682 & 133,00 \\
\hline Mixto maligno & 3 & 1,2 & $364(352-377)$ & $24,42(0,00-50,00)$ \\
\hline Tecoma maligno & 1 & 0,4 & 216 & 10,00 \\
\hline TOTAL Tumores Malignos & 86 & 34,8 & $373(350-395)$ & $170,82(90,22-251,42)$ \\
\hline Tumor funcional & 23 & 9,3 & $225(201-248)$ & $16,15(12,84-19,83)$ \\
\hline Fibrotecoma & 2 & 0,8 & $236(58-414)$ & $33,82(16,60-51,05)$ \\
\hline Cistoadenoma seroso & 57 & 23,1 & $244(233-256)$ & $22,97(10,42-35,51)$ \\
\hline Cistoadenoma mucinoso & 9 & 3,6 & $250(207-292)$ & $25,89(7,17-44,66)$ \\
\hline ATO & 5 & 2 & $269(217-321)$ & $45,32(30,65-59,98)$ \\
\hline Endometrioma & 22 & 8,9 & $245(218-273)$ & $49,01(30,92-67,11)$ \\
\hline Teratoma maduro & 34 & 13,8 & $239(221-258)$ & $14,65(11,03-18,51)$ \\
\hline Quiste gigantocelular & 1 & 0,4 & 379 & 5,70 \\
\hline Quiste de inclusión & 4 & 1,6 & $250(216-284)$ & $15,52(4,38-26,66)$ \\
\hline Neoplasia células esteroideas & 1 & 0,4 & 274 & 16,37 \\
\hline Tumor Sertoli-Leidying & 1 & 0,4 & 284 & 8,10 \\
\hline Tumor de Brenner & 2 & 0,8 & $226(0-452)$ & $17,05(6,10-28,00)$ \\
\hline TOTAL Tumores Benignos (n) & 161 & 65,2 & $243(235-251)$ & $24,29(18,87-29,71)$ \\
\hline TODOS los Tumores & 247 & 100 & $288(276-300)$ & $75,31(46,05-104,57)$ \\
\hline
\end{tabular}

TBPM: Tumor con bajo potencial de malignidad; ATO: absceso tuboovárico. $\bar{x}$ : media. IC95\%: intervalo de confianza 95\%. Recuento plaquetario/nl. CA-125 U/ml. 
Tabla 2. Distribución de tumores malignos según histopatología, estadio y medias de plaquetas y de CA-125

\begin{tabular}{|c|c|c|c|}
\hline TUMORES MALIGNOS & Frecuencia $(\%)$ & $\overline{\mathrm{x}}$ plaquetas (IC95\%) & x CA-125 (IC95\%) \\
\hline Estadio I & $25(29,07)$ & $326(286-366)$ & $66,43(29,97-102,89)$ \\
\hline Estadio II & $9(10,46)$ & $350(299-400)$ & $115,32(110,01-221,31)$ \\
\hline Estadio III & $38(44,18)$ & $413(381-445)$ & $251,96(79,94-423,98)$ \\
\hline Estadio IV & $8(9,3)$ & $442(350-534)$ & $288,97(26,53-551,41)$ \\
\hline Estadios I y II & $34(39,53)$ & $332(301-361)$ & $79,37(44,92-123,08)$ \\
\hline Estadios III y IV & $46(53,48)$ & $418(387-452)$ & $258,40(150,10-413,77)$ \\
\hline ТВРM & $6(6,97)$ & $237(198-276)$ & $17,28 \quad(3,53-31,03)$ \\
\hline Epiteliales malignos (todos) & $76(88,37)$ & $374(351-397)$ & $186,51(95,87-277,16)$ \\
\hline Premenopausia & $35(40,7)$ & $382(349-415)$ & $142,12(75,01-209,22)$ \\
\hline Posmenopausia & $41(47,67)$ & $367(333-401)$ & $224,41(63,66-385,17)$ \\
\hline Epiteliales malignos (sin TBPM) & $70(81,39)$ & $386(362-409)$ & $201,02(103,20-298,84)$ \\
\hline Premenopausia & $31(36,04)$ & $403(373-432)$ & $157,79(83,52-232,06)$ \\
\hline Posmenopausia & $39(45,35)$ & $372(337-403)$ & $235,38(66,78-403,98)$ \\
\hline No Epiteliales malignos & $10(11,62)$ & $362(264-458)$ & $51,53(1,29-101,76)$ \\
\hline Premenopausia & $10(11,62)$ & $362(264-458)$ & $51,53(1,29-101,76)$ \\
\hline Posmenopausia & $0(0)$ & - & - \\
\hline TOTAL Tumores Malignos & $86(100)$ & $373(350-395)$ & $170,82(90,22-251,42)$ \\
\hline
\end{tabular}

TBPM: tumores con bajo potencial de malignidad. x: media. IC95\%: intervalo de confianza $95 \%$. Recuento plaquetario / $\mathrm{nl}$. CA-125 U/ml.
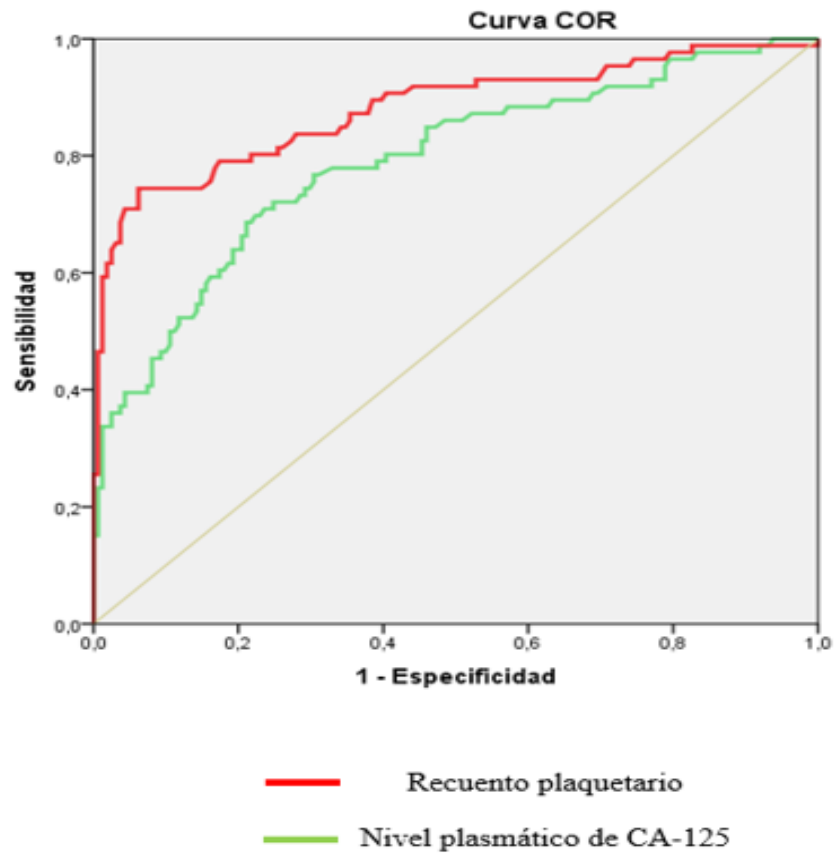

Linea de referencia

Figura 2. Curvas ROC de recuento plaquetario y concentración de CA-125 en todas las pacientes estudiadas. 
Tabla 3. Rendimiento diagnóstico de CA-125 y recuento plaquetario.

\begin{tabular}{lcccccccccc}
\hline Variable & AUC (IC95 \%) & S (\%) & E (\%) & VPP (\%) & VPN (\%) & ED (\%) & LR+ & LR- & DOR & $X^{2}$ \\
\hline Todas & & & & & & & & & & \\
CA-125 & $0,790(0,729-0,851)$ & & & & & & & & & \\
$\geq 35$ & & 61,62 & 81,36 & 63,85 & 79,87 & 74,49 & 3,3 & 0,47 & 7,01 & $<0,001$ \\
Plaquetas & $0,880(0,829-0,930)$ & & & & & & & & & \\
$>350$ & & 59,3 & 98,13 & 94,44 & 81,86 & 84,61 & 31,71 & 0,41 & 77,09 & $<0,001$ \\
$>400$ & 29,06 & 99,37 & 96,15 & 72,39 & 74,89 & 46,12 & 0,71 & 66,09 & $<0,001$ \\
Premenopausia & & & & & & & & & & \\
CA-125 & $0,732(0,640-0,823)$ & & & & & & & & & \\
$\geq 35$ & & 55,55 & 78,68 & 49,01 & 82,75 & 72,45 & 2,6 & 0,56 & 4,61 & $<0,001$ \\
Plaquetas & $0,873(0,802-0,944)$ & & & & & & & & & \\
$>350$ & & 64,44 & 98,36 & 93,54 & 88,23 & 89,22 & 39,29 & 0,36 & 109,18 & $<0,001$ \\
$>400$ & 31,11 & 99,18 & 93,33 & 79,6 & 80.83 & 37,93 & 0,69 & 55,07 & $<0,001$ \\
Posmenopausia & & & & & & & & & & \\
CA-125 & $0,872(0,792-0,953)$ & & & & & & & & & \\
$\geq 35$ & & 68,29 & 89,74 & 87,5 & 72,91 & 78,75 & 6,65 & 0,35 & 18,85 & $<0,001$ \\
Plaquetas & $0,907(0,838-0,976)$ & & & & & & & & & \\
$>350$ & & 53,65 & 97,43 & 95,65 & 66,66 & 75 & 20,87 & 0,47 & 44,02 & $<0,001$ \\
$>400$ & & 26,82 & 100 & 100 & 56,52 & 62,5 & - & 0,73 & - & $<0,001$ \\
\hline
\end{tabular}

Recuento plaquetario/nl. CA-125 U/ml. Área bajo la curva (AUC). Sensibilidad (S), especificidad (E), valor predictivo positivo (VPP), valor predictivo negativo (VPN), exactitud diagnóstica (ED), likelihood ratio positivo (LR+), likelihood ratio negativo (LR-) y Odds ratio diagnóstica (DOR).

\section{DISCUSIÓN}

La edad media en tumores epiteliales malignos fue menor a reportes estadounidenses (60-65 años) (19). Esta diferencia puede deberse a la inclusión de los TBPM como cáncer, que se presentan en promedio 1520 años antes $(20,21)$ y a que las mujeres latinas tienen características reproductivas diferentes a mujeres del hemisferio norte $(22,23)$. Hubo concordancia en la edad media de tumores malignos de origen germinal (24).

Un rendimiento más discreto de CA-125 en este análisis puede deberse a una menor prevalencia de estadios avanzados en la serie (53,48 \% vs 58,8 \%) (14), siendo estas etapas de la enfermedad las que más elevan este marcador (9). Debe considerarse también, que esta serie presentó una prevalencia menor a lo descrito de cáncer epitelial ovárico tipo II según carcinogénesis, los cuales son más agresivos y tienen mayor capacidad diseminadora, como se ha descrito (25).

En la serie estudiada, la media del recuento plaquetario fue notoriamente mayor en tumores malignos frente a los benignos, siendo una de las excepciones los TBPM. Este hallazgo es similar a lo reportado recientemente (15). Otras excepciones fueron el carcinoma de Brenner, neuroendocrino carcinoide y el tecoma maligno. Esto sugiere que estirpes raras pudiesen presentar un comportamiento en recuento plaquetario similar a tumores benignos y TBPM, sin embargo, son necesarias series con mayor número de estos tumores para confirmar esta sospecha. Por otra parte, tanto esta serie como estudios recientes observaron que en 
estadios FIGO más avanzados también es mayor el recuento plaquetario y de CA-125 $(14,15)$.

Al evaluar independientemente al CA-125 y el recuento plaquetario no se observó diferencias estadísticamente significativas con base en un intervalo de confianza (IC) $95 \%$ del área bajo la curva, lo que sugiere, por lo menos en esta serie, que son de utilidad similar. Un estudio (15) también retrospectivo que mostró rendimiento similar, pero con discreta mayor utilidad de CA-125 sobre el conteo de plaquetas, pareciera presentar mayor sesgo de selección al solo estudiar tumores de alto riesgo y con el doble de prevalencia para malignidad. A menor predominio de malignos en una serie, los resultados son más representativos para enfrentar cotidianamente los tumores de ovario en población no seleccionada, donde la prevalencia es menor al $1 \%$ (26-28). Es bien conocido que, al evaluar la eficacia diagnóstica de una prueba, el resultado de positivo o negativo de likelihood ratio (LR+ LR-) no depende de la prevalencia de la enfermedad $(28,29)$, por lo que serían más confiables los valores de $\mathrm{LR}+\mathrm{y}$ LR- $=31,7$ y 0,41 en este análisis vs LR + y LR- $=2,61$ y 0,72 en Reino Unido (15).

Los tumores mucinosos presentan mayor dificultad diagnóstica al hacer el corte congelado porque usualmente son de mayor volumen y pueden presentar áreas benignas, borderline y malignas en el mismo tumor que al final será clasificado y tratado de acuerdo con el componente más atípico de los que presente (30, 31). Llamó la atención que estos tumores presentaron similar comportamiento de CA-125 tanto benignos como malignos invasivos, pero los adenocarcinomas de este tipo mostraron niveles elevados de recuento plaquetario con media para trombocitosis, a diferencia de los benignos y borderline. Por otro lado, incluso en ecografías por operadores expertos, es difícil clasificar los tumores mucinosos específicamente como benignos, borderline o malignos invasivos por sus ecopatrones compartidos $(32,33)$. Sería interesante contar con estudios de series grandes y multicéntricos que combinen características clínicas, ecográficas y hematológicas para tratar de aproximar el diagnóstico en estos tumores antes del reporte anatomopatológico final.

Al igual que reportes previos (34), en esta serie los abscesos tuboováricos (ATO) y endometriomas presentaron cifras de CA-125 elevadas similares a las de tumores malignos. En este sentido, un aporte de esta investigación fue mostrar que los recuentos plaquetarios en ATO y endometriomas fueron similares a los benignos. La ecografía ejecutada por operador experimentado y/o entrenado para discriminar masas anexiales es el método diagnóstico preoperatorio que mejor rendimiento ha mostrado al diferenciar masas benignas de malignas $(35,36)$. Sin embargo, estudios con operadores entrenados y experimentados en ecografía de masas anexiales han mostrado ATO como falsos positivos para malignidad en sus tablas descriptivas, debido a ser lesiones no homogéneas, tamaño aumentado, con componente sólido, doppler grado 3-4 (moderado a severo) sugiriendo neovascularización y muchas veces sin ecopatrón típico descrito (36-39). Aunque las características clínicas de dolor y edad ayudarían a la diferenciación de ATO y endometriomas con ecopatrón no frecuente, la duda diagnóstica puede persistir. El recuento plaquetario $\leq 350 / \mathrm{nl}$ pudiese aportar un dato adicional para clasificar los ATO como masas benignas sobre todo en aquellos que se presentan como masas ecográficamente sospechosas de malignidad + CA125 elevado cuando se tengan dudas, pero es necesario series más numerosas que permitan estrechar IC $95 \%$ proporcionando resultados estadísticamente más sólidos.

En contraste con lo previamente reportado (9), todas las AUC fueron mayores para recuento plaquetario versus concentración de CA-125 (figura 2 y tabla 3), pero con intervalos de confianza que sugieren una utilidad 
similar de ambos marcadores. El mejor rendimiento diagnóstico se demostró en mujeres posmenopáusicas con muy altos valores de E y VPP, no así para CA-125. Esto es relevante considerando que esta etapa de la vida concentra el mayor número de cánceres ováricos. No se encontraron publicaciones que analizaran el recuento plaquetario en tumores ováricos según estado menopáusico.

La mayor debilidad de este estudio es su carácter retrospectivo. Como fortaleza el haberse realizado en un centro hospitalario de referencia oncológica ginecológica, con una prevalencia importante de tumores malignos, y análisis estadístico ampliado de media según tipo histológico y según estado menopáusico. Hasta donde se conoce es el primer estudio que se centra en recuento plaquetario para el diagnóstico de cáncer ovárico en todo el continente americano y la segunda serie más grande publicada mundialmente, siendo solo superado por estudio de Watrowski y cols. (14), en 2016.

Sin embargo, es necesario investigaciones similares que permitan observar si los datos obtenidos se replican en otras poblaciones y más aún con estudios prospectivos y multicéntricos, que se continúen con metaanálisis y así justificar o no el uso del recuento plaquetario como un marcador de uso rutinario en la discriminación preoperatoria de tumores de ovarios.

Se concluye que el recuento plaquetario pudiera mejorar el rendimiento al combinarse con CA- 125 para diferenciar tumores ováricos malignos de benignos, pudiendo ayudar en la toma de decisiones en estas pacientes. Parece tener utilidad para clasificar como verdaderos benignos algunas masas anexiales que generan dudas diagnósticas por sus altos niveles de CA-125 y características ecográficas, como por ejemplo ATO. Se sugiere considerar incluir el recuento plaquetario como componente de futuras fórmulas para cálculo del riesgo de malignidad en tumores de ovario junto a CA-125, datos clínicos y características ecográficas, considerando que es una prueba económica, ampliamente disponible y de uso rutinario preoperatorio en pacientes con tumores de ovario

\section{REFERENCIAS}

1. Carvalho JP, Moretti-Marques R, Filho ALDS. Adnexal mass: diagnosis and management. Rev Bras Ginecol Obstet. 2020; 42(7):438-43. doi: 10.1055/s-00401715547.

2. De Matías Martínez M, Noguero Meseguer MR, Cuesta Cuesta AB, Abreu Griego ED, Bartolomé Sotillos S, Martín de Los Ríos MD. [Application of a protocol for the management of adnexal masses: savings in clinically unnecessary activity and costs]. An Sist Sanit Navar. 2020; 43(2):151-157. Spanish. doi: 10.23938/ ASSN.0863.

3. Díaz L, Omaña D, Santos M, Zambrano B. ¿Se justifica la ooforectomía profiláctica en histerectomías? Rev Obstet Ginecol Venez [Internet]. 2009 [consultado 22 de marzo de 2021]; 69(4):231-238. Disponible en:http://ve.scielo.org/scielo.php?script $=$ sci arttext\&pid=S0048-77322009000400004

4. Cancer Research UK [Internet]. London: Ovarian cancer survival statistics. One-, five- and ten-year survival for ovarian cancer. [consultado 22 de marzo 2021]. Disponible en: https://www.cancerresearchuk. org/health-professional/cancer-statistics/statistics-bycancer-type/ovarian-cancer/survival\#heading-Three.

5. Kalsi J, Gentry-Maharaj A, Ryan A, Singh N, Burnell $\mathrm{M}$, Massingham S, et al. Performance Characteristics of the Ultrasound Strategy during Incidence Screening in the UK Collaborative Trial of Ovarian Cancer Screening (UKCTOCS). Cancers (Basel). 2021; 13 (4): 858. doi: 10.3390/cancers 13040858 .

6. Bottsford-Miller J, Choi HJ, Dalton HJ, Stone RL, Cho MS, Haemmerle M, et al. Differential platelet levels affect response to taxane-based therapy in ovarian cancer. Clin Cancer Res. 2015; 21 (3): 602-10. doi: 10.1158/1078-0432.CCR-14-0870.

7. Orellana R, Kato S, Erices R, Bravo ML, Gonzalez P, Oliva $\mathrm{B}$, et al. Platelets enhance tissue factor protein and metastasis initiating cell markers, and act as chemoattractants increasing the migration of ovarian cancer cells. BMC Cancer. 2015; 15:290. doi: 10.1186/ s12885-015-1304-z. 
8. Feng S, Kroll MH, Nick AM, Sood AK, AfsharKharghan V. Platelets are not hyperreactive in patients with ovarian cancer. Platelets. 2016; 27(7):716-718. doi: 10.3109/09537104.2016.1174204.

9. Chalas E, Welshinger M, Engellener W, Chumas J, Barbieri R, Mann WJ. The clinical significance of thrombocytosis in women presenting with a pelvic mass. Am J Obstet Gynecol. 1992; 166 (3):974-977. doi: 10.1016/0002-9378(92)91375-k.

10. Kerpsack JT, Finan MA. Thrombocytosis as a predictor of malignancy in women with a pelvic mass. J Reprod Med [Internet]. 2000 [consultado 22 de marzo de 2021]; 45(11):929-932. Disponible en: https://www.researchgate.net/publication/12202007 Thrombocytosis_as_a_predictor_of_malignancy_in_women_with_a_pelvic_mass

11. Haddad LB, Laufer MR. Thrombocytosis associated with malignant ovarian lesions within a pediatric/ adolescent population. J Pediatr Adolesc Gynecol. 2008; 21(5):243-246. doi: 10.1016/j.jpag.2007.09.004.

12. Al-Nakaash N, Al-Hasan MA, Ghazi W. Thrombocytosis as a Predictor of Malignancy in Patients with a Pelvic Mass. Iraqi J Comm Med [Internet]. 2008 [consultado 22 de marzo 2021]; 21 (2):115-119. Disponible en: https://www.iasj.net/iasj/download/bde99a8a4c209743.

13. Atacag T. Diagnostic value of thrombocytosis and high CA 125 level in women with adnexal masses. Eur J Gynaecol Oncol [Internet]. 2012 [consultado 22 de marzo de 2021]; 33(5):517-520. Disponible en: https://www.researchgate.net/publication/233786758 Diagnostic_value_of_thrombocytosis_and_high CA_125_level_in_women_with_adnexal_masses

14. Watrowski R, Heinze G, Jäger $\bar{C}$, Forster J, Zeillinger R. Usefulness of the preoperative platelet count in the diagnosis of adnexal tumors. Tumour Biol. 2016; 37(9):12079-12087. doi: 10.1007/s13277-016-5090-2.

15. Abdulrahman GO, Das N, Lutchman Singh K. The predictive role of thrombocytosis in benign, borderline and malignant ovarian tumors. Platelets. 2020; 31(6):795-800. doi: 10.1080/09537104.2019.1686755.

16. Díaz L, Santos M, Zambrano B, Omaña D, Carrocci A. Tumores de ovario: índices de riesgo de malignidad y modelo ADNEX de IOTA. Opciones diagnósticas sin tecnología Doppler. Rev Obstet Ginecol Venez [Internet]. 2017 [consultado 22 de marzo 2021]; 77 (3):181-195. Disponible en: http://www.sogvzla. org.ve/sogvzla $20186 / \mathrm{cms} / \mathrm{svcobtenerpdfrevista.}$ php? $\mathrm{id}=0000000086 \&$ tipo $=$ normal\&fila $=6$

17. Zambrano B, Santos M, Díaz L, Omaña D. Experiencia en el tratamiento del cáncer ovárico avanzado. Rev Venez Oncol [Internet]. 2017 [consultado 22 de marzo de 2021]; 29(4):232-243. Disponible en: http:// www.oncologia.org.ve/site/upload/revista/pdf/02. zambrano_b_(232-243).pdf

18. Cohen JF, Korevaar DA, Altman DG, Bruns DE, Gatsonis CA, Hooft L, et al. STARD 2015 guidelines for reporting diagnostic accuracy studies: explanation and elaboration. BMJ Open. 2016; 6(11):e012799. doi: 10.1136/bmjopen-2016-012799.

19. Yancik R, Ries LG, Yates JW. Ovarian cancer in the elderly: an analysis of Surveillance, Epidemiology, and End Results Program data. Am J Obstet Gynecol. 1986; 154(3):639-647. doi: 10.1016/0002-9378(86)90623-x.

20. Sayasneh A, Ferrara L, De Cock B, Saso S, Al-Memar $\mathrm{M}$, Johnson S, et al. Evaluating the risk of ovarian cancer before surgery using the ADNEX model: a multicentre external validation study. Br J Cancer. 2016; 115(5):542548. doi: 10.1038/bjc.2016.227.

21. Chen YN, Ma F, Zhang YD, Chen L, Li CY, Gong SP. Ultrasound Features Improve Diagnostic Performance of Ovarian Cancer Predictors in Distinguishing Benign and Malignant Ovarian Tumors. Curr Med Sci. 2020; 40(1):184-191. doi: 10.1007/s11596-020-2163-7.

22. Henderson KD, Bernstein L, Henderson B, Kolonel L, Pike MC. Predictors of the timing of natural menopause in the Multiethnic Cohort Study. Am J Epidemiol. 2008; 167(11):1287- 1294. doi: 10.1093/aje/kwn046.

23. Forman MR, Mangini LD, Thelus-Jean R, Hayward MD. Life-course origins of the ages at menarche and menopause. Adolesc Health Med Ther. 2013; 4:1-21. doi: 10.2147/AHMT.S15946.

24. Guillem V, Poveda A. Germ cell tumours of the ovary. Clin Transl Oncol. 2007; 9(4):237- 243. doi: 10.1007/ s12094-007-0045-0.

25. Kurman RJ, Shih IeM. The Dualistic Model of Ovarian Carcinogenesis: Revisited, Revised, and Expanded. Am J Pathol. 2016; 186(4):733-747. doi: 10.1016/j. ajpath.2015.11.011.

26. Smith-Bindman R, Poder L, Johnson E, Miglioretti DL. Risk of Malignant Ovarian Cancer Based on Ultrasonography Findings in a Large Unselected Population. JAMA Intern Med. 2019; 179(1):71-77. doi: 10.1001/jamainternmed.2018.5113.

27. Parazzini F, Frattaruolo MP, Chiaffarino F, Dridi D, Roncella E, Vercellini P. The limited oncogenic potential of unilocular adnexal cysts: A systematic review and meta-analysis. Eur J Obstet Gynecol Reprod Biol. 2018; 225:101-109. doi: 10.1016/j.ejogrb.2018.04.019. 
28. Avilés G, Vaccaro H, Díaz L, Amor F, Iturra A, León M, et al. Curva de Aprendizaje para el Diagnóstico Ecográfico Específico de Masas Anexiales. Rev Chil Obstet Ginecol [Internet]. 2021 [consultado 22 de marzo 2021]; 86(2):137-151. Disponible en: https://www.scielo.cl/ pdf/rchog/v86n2/0717-7526-rchog-86-02-0137.pdf.

29. Eusebi P. Diagnostic accuracy measures. Cerebrovasc Dis. 2013; 36(4):267-272. doi: 10.1159/000353863.

30. Md Arshad NZ, Ng BK, Md Paiman NA, Abdullah Mahdy Z, Mohd Noor R. Intra-Operative Frozen Sections for Ovarian Tumors - A Tertiary Center Experience. Asian Pac J Cancer Prev. 2018; 19(1):213218. doi: 10.22034/APJCP.2018.19.1.213.

31. Zhang W, Jia S, Xiang Y, Yang J, Jia C, Leng J. Factors associated with misdiagnosis of frozen section of mucinous borderline ovarian tumor. J Int Med Res. 2019; 47(1):96-104. doi: 10.1177/0300060518795582.

32. Pascual A, Guerriero S, Rams N, Juez L, Ajossa S, Graupera B, et al. Clinical and ultrasound features of benign, borderline, and malignant invasive mucinous ovarian tumors. Eur J Gynaecol Oncol. 2017; 38(3):382386. doi: 10.12892/ejgo3560.2017

33. Moro F, Zannoni GF, Arciuolo D, Pasciuto T, Amoroso $\mathrm{S}$, Mascilini $\mathrm{F}$, et al. Imaging in gynecological disease (11): clinical and ultrasound features of mucinous ovarian tumors. Ultrasound Obstet Gynecol. 2017; 50 (2):261-270. doi: 10.1002/uog.17222.

34. Dolgun ZN, Kabaca C, Karateke A, İyibozkurt C, İnan C, Altıntaş AS, et al. The Use of Human Epididymis 4 and Cancer Antigen 125 Tumor Markers in the Benign or Malignant Differential Diagnosis of Pelvic or Adnexal Masses. Balkan Med J. 2017; 34(2):156-162. doi: 10.4274/balkanmedj.2016.0223.
35. Meys EM, Kaijser J, Kruitwagen RF, Slangen BF, Van Calster B, Aertgeerts B, et al. Subjective assessment versus ultrasound models to diagnose ovarian cancer: A systematic review and meta-analysis. Eur J Cancer. 2016; 58:17-29. doi: 10.1016/j.ejca.2016.01.007.

36. Valentin L, Jurkovic D, Van Calster B, Testa A, Van Holsbeke C, Bourne T, et al. Adding a single CA 125 measurement to ultrasound imaging performed by an experienced examiner does not improve preoperative discrimination between benign and malignant adnexal masses. Ultrasound Obstet Gynecol. 2009; 34(3):345354. doi: 10.1002/uog.6415.

37. Alcázar JL, Díaz L, Flórez P, Guerriero S, Jurado M. Intensive training program for ultrasound diagnosis of adnexal masses: protocol and preliminary results. Ultrasound Obstet Gynecol. 2013; 42(2):218-23. doi: 10.1002/uog.12440.

38. Díaz L, Zambrano B, Adami FJ, Alcázar JL. External Validation of Gynecological Imaging and Reporting Data System for Sonographic Evaluation of Adnexal Masses. Donald School J Ultrasound Obstet Gynecol. 2017; 11(2):135-140. Doi: 10.5005/jp-journals-10009-1514

39. Hidalgo JJ, Ros F, Aubá M, Errasti T, Olartecoechea B, Ruiz-Zambrana Á, et al. Prospective external validation of IOTA three-step strategy for characterizing and classifying adnexal masses and retrospective assessment of alternative two-step strategy using simple-rules risk. Ultrasound Obstet Gynecol. 2019; 53(5):693-700. doi: 10.1002/uog.20163. 